\title{
Multiresistente gramnegative Bakterien - die neuen Empfehlungen
}

\author{
Elisabeth Meyer
}

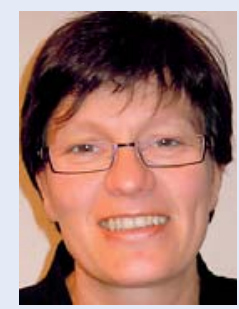

Elisabeth Meyer
$\mathrm{W}$ as tun bei Patienten mit ESBL oder generell bei Patienten mit multiresistenten Erregern? Isolieren oder nicht?

Fast jedes Krankenhaus hatte sich in der Vergangenheit ein eigenes Vorgehen zurecht gelegt: in einer Umfrage unter 359 Krankenhäusern, die am KrankenhausInfektions-Surveillance-System (KISS) teilnehmen, gaben 30\% der Kliniken an, ESBL-Patienten immer im Einzelzimmer zu isolieren, 58\% wenn möglich, während $12 \%$ sagten, dass sie für ESBL-Patienten keine Einzelzimmerisolierung verlangten. Ähnlich unterschiedlich war das Vorgehen beim Screening: etwa dreiviertel aller Teilnehmer screenten Patienten nicht auf ESBL, etwa ein fünftel nur definierte Risikopatienten; demgegenüber screenten $5 \%$ alle aufgenommenen Patienten.

Wenn man als Mitarbeiter oder Patient in ein anderes Krankenhaus wechselte, konnte es einem passieren, dass dort ein völlig anderes Hygieneregime herrschte und mitunter auch vehement vertreten wurde. Das führte zu Verunsicherung. Mein persönlicher Eindruck war immer, dass sich die Transmissionsraten von Hardliner-Kliniken (immer alle Patienten isolieren) und Nicht-Hardliner-Klinken (Standardhygiene, nicht isolieren) nicht groß unterschieden. Systematisch untersucht wurde dies nicht.

Multiresistente gramnegative Erreger sind, im Gegensatz zu MRSA, weltweit im Ansteigen begriffen. Viele Patienten kommen kolonisiert ins Krankenhaus. Multiresistente gramnegative Erreger werden überwiegend ins Krankenhaus importiert. In der öffentlichen Wahrnehmung verhält es sich jedoch anders; dort werden resistente Erreger gleichgesetzt mit „Krankenhauskeimen“ oder noch medienwirksamer mit „Killerkeimen aus dem Krankenhaus“. Das Reservoir, zumindest bei E. coli, liegt aber überwiegend im ambulanten Bereich. Dazu zwei Untersuchungen. 2011 wurden Hygienemitarbeiter bei einer Tagung gebeten, anonym einen Rektalabstrich abzugeben: unter 231 Teilnehmer wurde bei 3,5\% ein ESBL-E. coli gefunden. Noch höher, nämlich bei 6,7\% lag die Prävalenz von ESBL im Rahmen einer Untersuchung vom Landesamt für Gesundheit und Lebensmittelsicherheit (LGL) Bayern. Das LGL hatte über 3000 gesunde Familienmitglieder von Patienten, die im Lebensmittelbereich arbeiteten und vom LGL nachuntersucht wurden, gescreent. Wir können also davon ausgehen, dass etwa 5\% der Menschen in Deutschland ESBL- E.coli in sich tragen.

Das Problem der multiresistenten gramnegativen Bakterien nimmt weiter zu, die Ursachen sind vielschichtig, sie liegen nicht primär im stationären Bereich und das Vorgehen der Krankenhäuser ist entsprechend uneinheitlich.

Deshalb hat die Kommission für Krankenhaushygiene und Infektionsprävention (KRINKO) am Robert-Koch Institut neue Empfehlungen für den Umgang mit multiresistenten gramnegativen Bakterien erarbeitet und veröffentlicht. Diese sind in diesem Heft detailliert auf Seite 274 im Artikel „Neue KRINKO-Empfehlung Hygienemaßnahmen bei Infektionen oder Besiedlung mit multiresistenten gramnegativen Stäbchen“ vorgestellt.

Für viele Kliniken ist damit vieles einfacher geworden vorausgesetzt, dass die mikrobiologischen Labore die Befunde entsprechend kennzeichnen (als 3MRGN oder 4MRGN). Viele Isolierungen auf sog. Normalstationen sind wahrscheinlich nicht notwendig; die Evidenz dafür ist allerdings - wie Dr. Just in seinem Artikel richtig schreibt - mäßig. Auch wenn RKI-Empfehlungen durch die Änderung des Infektionsschutzgesetzes einen höheren Verbindlichkeitscharakter haben, sind sie nicht in Stein gemeißelt, sondern müssen sich in der Praxis bewähren. Ich habe allerdings keinen Zweifel, dass sie das tun werden.

Nicht den multiresistenten Bakterien, aber Ihnen alles Gute für das Neue Jahr wünscht

Elisabeth Meyer 


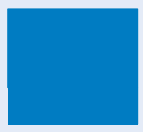

